\title{
THE COLORS OF COLLOIDS. V V
}

\author{
BY WILDER D. BANCROFT
}

\section{Metallic and Vitreous Lustre}

It has been stated already that whenever light in one medium falls upon the surface separating it from another medium some of the light is reflected within the first, while the remainder passes out into the second medium, except when the first is of lower refractivity than the second and the light falls at an angle greater than that of total reflection. Similarly, when light impinges upon a cut stone, some of it is reflected and the remainder passes into the stone. What is the relative amount of reflected light depends upon the nature of the stone-its refractivity and hardness-and determines its lustre; the greater the amount the more lustrous will the stone appear. "There are different kinds of lustre, and the intensity of each depends on the polish of the surface. From a dull, $i . e$. , an uneven surface the reflected light is scattered, and there are no brilliant reflections. All gem-stones take a good polish, and therefore have considerable brilliancy, so long as the surface retains its polish; turquoise, on account of its softness, is always comparatively dull.

"The different kinds of luster are:

"(I) Adamantine, characteristic of diamond.

"(2) Vitreous, as seen on the surface of fractured glass.

"(3) Resinous, as shown by resins.

"Zircon and demantoid, the green garnet called by jewelers 'olivine,' alone among gem-stones have a lustre approaching that of diamond. The remainder all have a vitreous lustre, though varying in degree, the harder and the more refractive species being on the whole the more lustrous.

"Some stones-for instance, a cinnamon garnet-appear to have a certain greasiness in the lustre, which is caused by stray reflections from inclusions or other breaks in the homogeneity of the interior. A pearly lustre, which arises from 
cleavage cracks and is typically displayed by the cleavage face of the topaz, would be seen in a cut stone only when flawed. Certain corundums when viewed in the direction of the crystallographic axis display six narrow lines of light radiating at angles of $60^{\circ}$ from a center in a manner suggestive of the conventional representations of stars. Such stones are consequently known as asterias, or more usually star-stonesstar-rubies or star-sapphires, as the case may be, and the phenomenon is called asterism. These stones have not a homogeneous structure, but contain tube-like cavities regularly arranged at angles to the crystallographical axis."

"Chatoyancy is a somewhat similar phenomenon, but in this case the fibers or cavities are parallel to a single direction, and a single broadish band is displayed at right angles to it. Cat's eyes as these stones are termed are cut en cabochon parallel to the fibers. The true cat's eye is a variety of chrysoberyl, but the term is also often applied to quartz showing a similar appearance. The latter is really a fibrous mineral, such as asbestos, which has become converted into silica. The beautiful tiger's eye from South Africa is a silicified crocidolite, the original blue color of which has been altered by oxidation to golden brown. Recently tourmalines have been discovered which are sufficiently fibrous in structure to display an effective chatoyancy.

"The milky sheen of moonstone owes its effect to reflections from twin lamellae. The wonderful iridescence which is the glory of the opal, and is therefore termed opalescence, arises from a structure which is peculiar to that species. Opal is a solidified jelly; on cooling, it has become riddled with extremely thin cracks, which were subsequently filled with similar material of slightly different refractivity, and thus it consists of a series of films. At the surface of each film, interference of light takes place just as at the surface of a soapbubble, and the more evenly the films are spaced apart the more uniform is the color displayed, the actual tint depending upon the thickness of the films traversed by the light giving rise to the phenomenon." 
"Upon the fact that the refractive index of a substance varies for light of different colors depends such familiar phenomena as the splendor of the rainbow and the 'fire' of the diamond. When white light is refracted into a stone it no longer remains white, but is split up into a spectrum. Except in certain anomalous substances the refractive index increases progressively as the wave-length of the light decreases, and consequently a normal spectrum is violet at one end and passes through green and yellow to red at the other end. The width of the spectrum which may be measured by the difference between the refractive indices for the extreme red and violet rays also varies, though on the whole it increases with the refractive index. It is the dispersion, as this difference is termed, that determines the 'fire'-a character of the utmost importance in colorless transparent stones, which, but for it, would be lacking in interest. Diamond excels all colorless stones in this respect, although it is followed closely by zircon, the color of which has been driven off by heating; it is, however, surpassed by two colored species: sphene which is seldom seen in jewelery, and demantoid, the green garnet from the Urals, which often passes under the misnomer olivine."

"Alexandrite, a variety of chrysoberyl, is a curious and instructive case. The balance in the spectrum of light transmitted through it is such that, whereas in daylight such stones appear green, in artificial light, especially in gas-light they are a pronounced raspberry red. The phenomenon is intensified by the strong dichroism characteristic of this species."

"Any mollusc, whether of the bi-valve or the uni-valve type, which possesses a nacreous shell, has the power of producing pearls; but only two, the pearl oyster, Meleagrina. margaretifera, and the pearl mussel, Unio margarifer, repay the cost of systematic fishing. The outside of the shell is formed of the horny matter called conchiolin; while the inside is composed of two coats of which the outer consists of alternate layers of conchiolin and calcium carbonate in its crystallized form, calcite, and the inner of the same organic matter, but with calcium carbonate in its other crystallized form, 
aragonite. The latter coat forms the nacreous lining known as mother-of-pearl, which is identical in consistency with pearl, but somewhat more transparent. The iridescence of motherof-pearl is due not only to the fact that it is composed of a succession of thin transparent layers; but also to the fact that these layers overlap like slates on a house, and form a series of fine parallel lines on the surface; diffraction therefore as well as interference of light takes place, and a similar diffraction phenomenon is displayed even by a cast of the inside of the shell. The animal has the property of secreting calcium carbonate which it absorbs from the sea-water, in both its crystallized conditions as well as conchiolin. At the outer rim it secretes conchiolin, further in calcite, and at the very inside aragonite. The shape and appearance of a pearl depend therefore on the position in which the intruding substance is situated within the shell."

"Built up in a haphazard manner and not confined by the inexorable laws of intermolecular action, a pearl may assume any and every variety of shape from the regular to the fantastic. It may be truly spherical, egg- or pear-shapedpear-drops or pear-eyes, as they are termed-or it may be quite irregular-the so-called baroque or barrok pearls. The first is the most prized, but a well-shaped drop pearl is in great demand for pendants or ear-rings. The color is ordinarily white, or faintly tinged yellowish or bluish, and somewhat rarely, salmon-pink, reddish, or blackish gray. Perfect black pearls are valuable, but not as costly as the finest of the white. Though not transparent, pearl is to a varying extent translucent, and its characteristic lustre--'orient' in the language of jewelery - is due to the same kind of interaction of light reflected from different layers that has been remarked upon in the case of opal and certain other stones. The translucency varies in degree, and some jewelers speak of the 'water' of pearls just as in the case of diamonds. If a pearl be sliced across the middle and the section be examined under the microscope; it will be seen that the structure consists of concentric shells and resembles that of an onion. Because of 
their composite character, pearls have a specific gravity ranging from 2.65 to $2.69-2.84-2.89$ in the case of pink pearls-which is appreciably less than that of aragonite: the hardness is about the same, namely 3.5 to 4 on Mohs' scale. That the arrangement of the mineral layers is approximately parallel is evinced by the distinctness of the shadow-edges shown on examination with the refractometer. Pearls require very careful handling because they are soft and therefore apt to be scratched, and because they are chemically affected by acids, and even by the perspiration from the skin. Acids attack only the calcium carbonate, not the organic matter; the well-known story therefore of Cleopatra dissolving a valuable pearl in vinegar, which is moreover too weak an acid to effect the solution, must not be accepted too literally. Pearls are not cut like stones, and therefore as soon as the precious bloom has once gone, nothing can be done to revive it. Attempts are sometimes made in the case of valuable pearls to remove the dull skin and lay bare another iridescent layer underneath, but the operation is exceedingly delicate. Even with the best of care pearls must in process of time perish owing to the decay of the organic constituent. Pearls that have been discovered in ancient tombs crumbled to dust at a touch, and those formerly in ancient rings have vanished or remain only as a brown powder, while the garnets or other stones set with them are little the worse for the centuries that have passed by."

Farrington ${ }^{1}$ differentiates more lustre by name, though he does not give any adequate definitions. "The lustre of gems is one of their most important and distinctive characters. Not only does it form one of the easiest means of distinguishing gems, but it is also one of the most reliable characters sought by those experts who depend for their determination of gems on ocular examination alone. One familiar with the lustre of quartz, as compared with that of diamond, for example, is in little danger of confusing the two, for the lustre of one can be recognized as adamantine, that of the other as

${ }^{1}$ Gems and Gem Minerals, 16, 2 19 (1903). 
vitreous. The lustre of a gem is produced by the light which it reflects back to the eye, and this may vary in quantity and quality with the nature of the surface. Since the latter is largely the result of the molecular structure of the mineral, it follows that different species will have distinctive lustre. The terms used to describe the different kinds of lustre are derived from that afforded by some well-known object. Thus adamantine lustre means the lustre of the diamond; vitreous lustre, the lustre of broken glass; oily lustre, the lustre of oil; waxy lustre, the lustre of wax; resinous lustre, the lustre of resins; pearly lustre, the lustre of pearl; silky lustre, the lustre of silk; and metallic lustre, the lustre of shining metals.

"Of the above kinds of lustre, the vitreous is the most common among gems, being displayed by quartz, topaz, beryl, tourmaline, sapphire, and many others. The adamantine lustre belongs almost exclusively to the diamond, although it is displayed to some extent by sphene and colorless zircon, and is suggested by some sapphires. It is characteristic of minerals of a high degree of refraction. Metallic lustre is strictly possessed only by opaque minerals, and hence among gems is confined to pyrite and hematite. The lustre of turquoise is of the waxy order. Essonite displays a somewhat resinous lustre, and chrysolite an oily one. Pearly lustre is best seen in the pearl, but is also illustrated by the moonstone and opal. Tiger eye and cat's eye afford examples of silky lustre."

"There are numerous ways of producing imitation pearls, one of which, invented many years ago by a French beadmaker named Jacquin, gives remarkably accurate reproductions. The Jacquin pearls are made from an easily fusible, bluish glass, which is first drawn into tubes, and, from these, hollow globules of the desired size are blown. These are covered on the inside with a solution of isinglass, and a substance called essence of pearl, which is blown in warm, and spread over the interior by rapid motion. When dry the globules are filled with wax. The essence of pearl, which constitutes the important feature of Jacquin's process, con- 
sists of a silvery substance found beneath the scales of the fish known as the bleak (Cyprinus alburmus). It is in the form of thin, irregular rhombic plates, and is obtained by washing the scales, one pound of essence being derived from seven pounds of scales. From eighteen thousand to twenty thousand fish are required to produce the latter amount of scales. The substance is, therefore, costly; and owing to this fact, and the amount of labor and skill required to make the pearls, they bring a considerable price. They can be distinguished from genuine pearls by their greater hardness, and a colder feeling in the hand."

The colors of mother-of-pearl have been studied by Pfund. " "A perpendicular section of the shell of a freshwater mussel, Abovaria ellipsis (Lea), taken near its growing edge shows that, speaking roughly, the shell may be said to consist of three layers:

"I. An outer horny layer (a) (periostracum) which is composed of organic matter (conchiolin).

"2. An intermediate layer (b) of minute prisms of $\mathrm{CaCO}_{3}$ separated by thin layers of conchiolin. This layer is termed the 'prismatic layer.'

"3. An inner layer (c) composed of alternate laminae of $\mathrm{CaCO}_{3}$ and conchiolin. This is the true mother-of-pearl or 'nacre.'

"That minute layers of conchiolin exist between those of $\mathrm{CaCO}_{3}$ is shown by placing a shell in hydrochloric acid. The calcium carbonate disappears, leaving a flabby, honeycombed mass of conchiolin in, practically, the form of the original shell.

"Whereas the minute layers of nacreous matter are continuous on the inside of the shell, they terminate abruptly on the outside. Each layer projects slightly beyond the edge of its predecessor, hence the outside of the nacreous portion will show innumerable steps or ridges running parallel to the growing edge of the shell. Such a surface structure is identical with that of a diffraction grating, hence, in case the spacing

${ }^{1}$ Jour. Franklin Inst., I83, 453 (I9I7). 
is sufficiently regular, diffraction colors might be looked for on the outside of a shell.

"In order to study these colors it is first necessary to grind off the horny and prismatic layers and polish the nacreous layers by means of a buffing wheel. By permitting the light from an incandescent lamp to be reflected from this surface, pure diffraction colors are observed. As a rule these colors are rather inconspicuous since they are masked by the flood of light coming from the underlying portions. These phenomena may be studied most readily by means of celluloid copies or replicas which are made by flowing celluloid dissolved in amyl acetate over the surface of the shell. After the amyl acetate has evaporated, the shell is immersed in water for about 20 minutes. The celluloid is then removed and dried on a plate of clear glass.

"A microphotograph obtained from such a replica was compared with a similar photograph of a replica taken from a Rowland diffraction grating having 7230 lines per inch. Since the scale of enlargement is the same in both cases it appears that the steps or ridges on the outside of the shell are spaced at the rate of about 9000 per inch. This particular shell belonged to a mussel taken from the Mississippi River. As might be expected replicas taken from the inside of the shell revealed no grating structure.

"It is obvious that the microscope may be dispensed with entirely in a study of the spacing of these lines for, by measuring the angle of diffraction of light of known wavelength, the grating space may be obtained at once. If a piece of cardboard with a pin-hole $0.5 \mathrm{~mm}$ in diameter be placed in contact with the replica and if a Nernst lamp be viewed through this opening, the two first order diffraction spectra may be seen. If the pin-hole be moved perpendicular to the direction of the lines a study may be made of the striking periodicities in the spacing of the lines. At intervals the lines will be spaced at the rate of 6-8000 lines per inch while the regions in between will show a spacing of $15^{-20,000}$ lines per inch. This is not the place to discuss the question as to how 
these layers are deposited by the living mussel or what the cause of the periodicity giving rise to layers of such remarkable uniformity is. Yet a study of these questions might be much facilitated by the use of replicas which, when viewed in the white light from a small, intense source, show extremely brilliant colors and color changes over the surface, thus enabling one to tell at a glance where the spacing is coarse or fine.

"Upon grinding the surface of mother-of-pearl with fine emery all traces of diffraction colors disappear and the brilliant iridescence alone remains. These colors can, obviously, be due only to interference in the parallel laminae. A rough test of this inference consists in reflecting light almost normally from a patch on the shell appearing red. By increasing the angle of incidence and moving the eye so as to receive always the reflected beam, the color changes in succession to orange, green, and finally blue. The same phenomenon is observed with Lippmann color-photographs and crystals of $\mathrm{KClO}_{3}$ whose structure is known to be laminar. In order to prove even more conclusively the correctness of this explanation, white light of great intensity was reflected from the motherof-pearl into a spectrometer and, by means of suitable apparatus, the intensity of the radiation in the near infra-red and visible spectrum was studied with the view of finding indications of interference maxima.....

"The maxima lie, respectively, at I.4 $\mu, 0.7 \mathrm{I} \mu$ and I. $86 \mu, 0.93 \mu$, and $0.63 \mu$. As before, the wave-lengths of successive maxima bear the ratios $I: 1 / 2: 1 / 3$ to a very fair degree of approximation-hence the conclusion may be drawn that the iridescent colors of mother-of-pearl owe their existence to interference of light reflected from the parallel and equidistant laminae.....

"Having investigated highly colored shells it seemed of interest to take up the study of nacreous matter which showed no color but a brilliant 'pearly' lustre. A suitable region on the inner surface of a Mississippi River mussel shell was found and from this a reflection curve shown was obtained. 
The lack of sharpness of the maxima indicates that the thickness of the laminae is far from being constant. It is possible to simulate the appearance of such a specimen by heating a piece of mica in a Bunsen flame. In consequence of the heating, the mica is split up into numerous laminae of widely varying thickness.

"An unsuccessful attempt was made to study, in a similar manner, that area on the outside of the shell which exhibits neither color nor pearly lustre. The character of the chalky material in the shell was such as to scatter the light diffuselythus preventing regular reflection from successive laminae. That laminae exist in this region also is certain, for the diffraction colors seen indicate a spacing of the laminae which is practically the same as that shown by neighboring iridescent portions."

The final conclusion is that mother-of-pearl owes its colors to the diffraction of light due to a grating-like structure of the terminal edges of successive laminae, and the interference of light due to reflection from numerous parallel laminae of sensibly equal thickness.

Pfund urges biologists to attack anew the problem of ascertaining the manner in which the layers of nacreous material are deposited. "The truly remarkable constancy of thickness of the laminae demands explanation. That periodic variations in external conditions such as light and darkness, tides, temperature, etc., should be responsible for the deposition of discrete layers seems improbable since the total number of laminae possessed by a shell of known age is entirely too great to be accounted for on any such hypothesis. Possibly the animal, as such, has nothing to do with the periodicity which gives rise to the laminae-for, just as the crystals in the minute laminae exhibited by $\mathrm{KClO}_{3}$ grow only in definite thickness, so may the minute crystals of $\mathrm{CaCO}_{3}$, surrounded by conchiolin, grow only until a certain thickness is reached."

Crookes ${ }^{1}$ says: "When a good fiery opal is examined in

${ }^{1}$ Proc. Roy. Soc., I7, 448 (1869). 
daylight, sunlight, or artificial light, it appears to emit vivid flashes of crimson, green, or blue light, according to the angle at which the incident light falls, and the relative position of the opal and the observer; for the direction of the path of the emitted beam bears no uniform proportion to the angle of the incident light. Examined more closely, the flashes of light are seen to proceed from planes or surfaces of irregular dimensions inside the stone, at different depths from the surface and at all angles to each other. Occasionally a plane emitting light of one color overlaps a plane emitting light of another color, the two colors becoming visible alternately upon slight variations of the angle of the stone; and sometimes a plane will be observed which emits crimson light at one end, changing to orange, yellow, green, etc., until the other end of the plane shines with a blue light, the whole forming a wonderfully beautiful solar spectrum in miniature. I need scarcely say that the colors are not due to the presence of any pigment, but are interference colors caused by minute striae or fissures lying in different planes. By turning the opal around and observing it from different directions, it is generally possible to get a position in which it shows no color whatever. Viewed by transmitted light, opals appear more or less deficient in transparency and have a slight greenish yellow or reddish tinge. In order to adapt them better to the purposes of the jeweler, opals are almost always polished with rounded surfaces, back and front; but the flashes of colored light are better seen and examined when the top and bottom of the gems are ground and polished flat and parallel. A good opal is not injured by moderate heating in water, soaking in turpentine, or heating strongly in Canada balsam and mounting as a microscopic slide....

"If an opal which emits a fine broad crimson light is held in front of the slit of a spectroscope or spectrum-microscope, at the proper angle, the light is generally seen to be purely homogeneous, and all the spectrum that is visible is a brilliant luminous line or band, varying somewhat in width and more or less irregular in outline, but very sharp, and 
shining brightly on a perfectly black ground. If now the source of light is moved so as to shine into the spectrumapparatus through the opal, the above appearance is reversed, and we have a luminous spectrum with a jet-black band in the red, identical in position, form of outline, and sharpness with the luminous band previously observed. If instead of moving the first source of light (the one which gave the reflected luminous line in the red), another source of light be used for obtaining the spectrum, the two appearances, of a colored line on a black ground and a black line on a colored ground, may be obtained simultaneously, and they will be seen to fit accurately. Those parts of the opal which emit red light are therefore seen to be opaque to light of the same refrangibility as that which they emit; and upon examining in the same manner other opals which shine with green, yellow or blue light, the same appearances are observed, showing that this rule holds good in these cases also. It is doubtless a general law, following of necessity the mode of production of the flashes of color."

When the transmission spectra are examined, they are found to consist of a black band, often spiral in shape, against a bright field, the band moving along the spectrum almost from one end to the other as the opal is moved sideways. "All these black bands can be reversed and changed into luminous bands by illuminating the opal with reflected light. They are, however, more difficult to see; for the colored light is only emitted at a particular angle, while the special opacity to the ray of the same refrangibility as the emitted ray holds good for all angles. It is scarcely necessary to say that the color of the moving luminous line varies with the part of the spectrum to which it belongs. The appearance of a luminous line, moving slowly across the black field of the instrument and assuming in turn all the colors of the spectrum, is very beautiful.

"The explanation of this phenomenon is probably as follows: In the case of the moving line, the light-emitting plane in the opal is somewhat broad, and has the property 
of giving out at one end, along its whole height and for a width equal to the breadth of the band, say, red light; this merges gradually into a space emitting orange, and so on throughout the entire length of the spectrum, or through that portion of it which is traversed by the moving line in the instrument, the successive pencils (or rather ribbons) of emitted light passing through all degrees of refrangibility. It is evident that if this opal is passed slowly across the slit of the spectrummicroscope, the slit will be illuminated successively with light of gradually increasing refrangibility, and the appearance of a moving luminous line will be produced; and if transmitted light is used for illumination, the reversal of the phenomenon will cause the production of a black line moving along a colored field. A diagonal line will be produced if an opal of this sort is examined in a sloping position. The phenomenon of a spiral line in relief rolling along as the opal is moved, is doubtless caused by modifying planes at different depths and connected by cross planes. I can form a mental picture of a structure which would produce this effect, but not clearly enough to enable me to describe it in words.

"It is probable that similar phenomena may be seen in many, if not all, bodies which reflect colored light after the manner of opals. A magnificent specimen of Lumacelli, or Fiery Limestone, from Italy, kindly presented to me by my friend David Forbes, shows two sharp, narrow and parallel bands in the red. I have also observed similar appearances in mother-of-pearl. The effects can be imitated to a certain extent by examining Newton's rings, formed between two plates of glass, in the spectrum instrument."

Madan ${ }^{1}$ considers that both the iridescent colors of potassium chlorate crystals and the colors of opals are due to the presence of prismatic cavities. "I may mention that plates of opal-at any rate those portions which give a uniform color-afford spectra extremely similar in character to those of the iridescent crystals; definite bright bands in the reflected light, and dark complementary bands in the transmitted light,

${ }^{1}$ Nature, 32, 102 (1885). 
changing their position in the spectrum with change of incidence. Now the iridescence of opal is pretty well known to be due to the reflection of light from the surfaces of rows of filaments embedded in the same. ${ }^{1}$ I cannot say that I have yet succeeded in actually bringing out such rows of tubes under the microscope. It is easy to see with $\mathrm{a} 1 / 5$ inch power, when the illuminating beam is properly adjusted, a sort of wavy structure at the twin-film (like that seen in opal under the same conditions); but I have not yet made out such definite rows of cavities as would seem to be required to produce the singularly uniform sheet of color. They may be beyond the power of a microscope altogether."

Stokes ${ }^{2}$ disagrees entirely with Madan's view. "The appearance of Mr. Madan's paper induces me to offer some additional remarks on this subject. In the discussion that followed the reading of my paper [on chlorate crystals] Mr. Crookes referred to the closely analogous spectra exhibited by opals, as described in his paper. This paper, though it came before me at the time when it was read, was not in my mind when I wrote my own. I called shortly afterwards at Mr. Crookes' house, and saw the spectra of his opals. Supposing that there were sufficient grounds for the commonly received idea that the colors of the opal are due to fine tubes in the mineral, we did not at the time conceive that the phenomena could be the same; were it not for this, I should certainly have added to my paper a reference to that of Mr. Crookes.

"Mr. Crookes was so good as to lend me his opals for more leisurely study. The further examination has so impressed me with the similarity of character of the spectra, that I am strongly disposed to think that the colors of the opal and those of the chlorate crystals may be due to the same cause. This does not, however, lead me to attribute tubes or striae to the chlorate crystals, the structure of which can

\footnotetext{
1 Brewster: Brit. Assoc. Reports, I844, II, 9.

2 Nature, 32, 224 (1885).
} 
comparatively easily be made out, but to doubt very greatly the theory which attributes the colors of opal to fine tubes.

"Mr. Madan does not profess to have actually seen in the chlorate crystals such tubes as he supposes to exist, nor could I see anything of the kind on examining some of the crystals I have got after the appearance of his paper. On the other hand, I notice that Brewster did not state that he had actually seen the supposed tubes, but merely inferred their existence from a comparison of the appearance under the microscope of the precious opal with that of hydrophane. And Mr. Crookes tells the that an opal is not spoiled or affected by being immersed in water or even oil. The fact is that it is extremely difficult to make out what the actual structure is with which we have to deal in the case of the opal, whereas in the case of the chlorate crystals it is unmistakable. Moreover, in the case of the chlorate crystals there is a wonderful uniformity in the phenomena presented by the same crystal, extending, it may be, over nearly the whole of even a large crystal, whereas in the opal the color extends over comparatively small patches; and even a single patch is seen under the microscope to present differences of structure in different parts. Hence if the colors in opal and those in the chlorate crystals are really due to a similar cause, it seems much more likely that a study of the phenomena of the chlorate crystals will throw light on those of the opal, than that the phenomena of the opal should furnish the key to the explanation of the colors of the chlorate crystals.

"In truth, I do not see how the presence of tubes, if such there be in the opal, would account for the phenomena, and especially for the very peculiar spectrum exhibited. The supposition of the existence of rows of tubes leads one to look in the direction of diffraction. But I do not see how monochromatic light, or, at least, light almost monochromatic, can be obtained by diffraction. And even independently of this consideration there is one feature of the production of color in the chlorate crystals which shows, at once and decisively, that at least in their case the color cannot be due to 
diffraction. If an iridescent crystal be chosen with an even surface, and the flame of a candle in a dark room be viewed by reflection in it, it is found that the color is seen in the direction of the regularly reflected light. In fact, the colored light forms a well-defined image of the flame of the candle, coinciding with, or overlapping, the colorless image due to reflection from the first surface. This differs altogether from what we get in the case of a grating, or in that of mother-of-pearl or Labrador spar. It agrees so far with the colors of thin plates, or the colors shown by reflection by certain quasi-metallic substances, such as several of the aniline dyes, though the production of color in these three cases is due to three totally different causes.

"It has been conclusively proved that the seat of the color in the chlorate of potash crystals is in a very thin twin stratum; and I entertain myself little or no doubt that the color depends in some way on the different orientation of the planes of polarization in the two components of a twin, and on the difference of retardation of the two polarized pencils which traverse the thin stratum. But anything beyond this is at present only a matter of speculation....

"In conclusion, I would mention an interesting paper on 'The Spectrum of the Noble Opal,' by Prof. H. Behrens, a copy of which I have just received by the kindness of the author. In this paper, which is printed in the Neues Jahrbuch fur Mineralogie, etc., 1873 , the author, who was evidently unacquainted with Mr. Crookes' paper when he wrote his own, has described and figured the peculiar spectra of several opals."

A glass surface from which light is totally reflected shines with a bright metallic lustre ${ }^{1}$ and "forms the clearest and most perfect mirror known to science. In the construction of optical instruments totally reflecting prisms are therefore used frequently to turn the rays of light in any desired direction."

This shows that the sensation of metallic lustre does not necessarily involve the presence of a metal or of metallic

1 Lommel: "Experimental Physics," 519 (190o). 
electrons, a fact which is also brought out very clearly by experiments of Dove ${ }^{1}$ and others on binocular lustre as it is sometimes called. Dove combined black and white figures stereoscopically and also colored figures obtaining the effect of metallic lustre in both cases although the single figures showed no lustre. "In one of two pictures for a stereoscope the section of a foreshortened pyramid was painted a saturated blue while the other was painted yellow. When green was seen as a result of stereoscopic combination it seemed as though the colors had become transparent and were seen one through the other. That many people do not see these colors combined but only alternately is due to the fact that [the accommodation for the two colors changes continually and that] these people are conscious only of the end-states and not of the intermediate ones. When a green color was seen, it seemed to me and to others that the color was covered with a varnish. This lustrous effect of the mixture was also noticed by Oertling when he combined in a stereoscope different colored sectors of a hemisphere painted like a balloon. These phenomena are not very striking and many people do not see them. On the other hand if one combines stereoscopically the yellow and blue sections of a pyramid while holding a violet glass before one eye, the image resembles a polished metallic surface, whereas the surface is matt when seen with either eye alone. Probably the violet glass brings the two colors to the same intensity.

"In all cases where a surface appears lustrous, there is always a specular, transparent or translucent layer of extreme thinness, through which one sees the other substance. The effect of lustre is therefore produced when light specularly reflected from the surface is combined with internally reflected or scattered light. The lustrousness increases with the number of alternations from one to another. Thus mica films have metallic lustre and a pile of glass plates the lustre of mother-of-pearl. The two sets 'of reflections act on the eye from different distances. If the eye adjusts itself so as

${ }^{1}$ Pogg. Ann., 83, I69 (I85 I). 
to see the substance behind the transparent film, the light that is reflected from the surface cannot be seen distinctly and the consciousness of a vaguely seen reflection gives the appearance of lustre. Lustre is therefore really a false or secondary phenomenon which dazzles and prevents us from seeing clearly the underlying surface. It disappears therefore if one eliminates the surface reflection by looking through a nicol prism at the varnish of a picture below the polarization angle. The interference phenomena due to the difference in phase of the light reflected from the two surfaces are therefore not the cause of the phenomenon of lustre but rather consequences of the conditions under which lustre occur."

Burckhardt ${ }^{1}$ points out that "instead of combining white and black in the stereoscope the eye can also do it by irradiation, short-sighted people far off and far-sighted people close up. When the black predominates in woolen goods striped black and white, these goods seem to me and to others perfectly lustrous exactly like the lustre of silk. Silk makers must have known this for a long time for they are very prone to increase the lustre of silk in this way."

Brewster $^{2}$ does not accept Dove's explanation of the phenomenon. "Some years ago it was observed by Professor Dove that when the right and left eye figures of a pyramid or other mathematical solid, the one drawn on a white and the other on a dark ground, were inserted in the spectroscope, the solid in relief appeared with a particular lustre. Prof. Dove described the lustre as metallic, and in another place, where he describes the two diagrams as drawn, the one with white lines on a black ground, and the other with black lines on a white ground, he stated that the pyramid in relief appears lustrous, as if made of graphite. Other observers described the lustres differently, some as resembling ground glass, and others as like paper darkened with a black lead pencil, while Professor Rood regarded it as recalling the idea of highly polished glass. In order to explain this phenomenon, Professor Dove re-

${ }^{1}$ Fortschritte der Physik, Io, 310 (1854).

2 Brit. Assoc. Reports, I86r, II, 29. 
marked that 'in every case where a surface appeared lustrous, there was always a transparent, or transparent-reflecting stratum of much intensity, through which we see another body. It is therefore externally reflected and dispersed light, whose combined action produced the idea of lustre. This effect, he elsewhere added, we see produced when many watchglasses are laid in a heap, or when a plate of transparent mica or talc, when heated red-hot, is separated into multitudes of thin layers, each of which, of inconceivable thinness, is found to be highly transparent, while the entire plate assumes the lustre of a plate of silver.' To these examples of lustre, produced by thin plates not in optical contact, or if in actual contact, having different reflective powers, were to be added the following: pearls, mother-of-pearl, pearl-spar, and composite crystals of calcareous spar, and decomposed glass of all colors. The cause of these various kinds of lustre, and of that of metals had already been well known, and when binocular lustre attracted the attention of philosophers, it was natural to ascribe it to the same cause. Professor Dove did this, and considered the dark surface in the one picture as the dispersed light, and the white surface as the regularly reflected light, the dark surface being seen through the white surface. This theory of binocular lustre, he had reason to believe, was not satisfactory.... In order to test this explanation by other means, he combined surfaces that had no geometrical figures upon them, and he found that binocular lustre was not produced. This experiment seemed decisive of the question. He was led to infer from it that the lustre observed in the combination of right and left eye figures of solids was not due to the rays from a dark surface passing through a lighter one to the eye, but to the effect of the eyes in combining the two stereoscopic figures, and to the dazzle occasioned by the alternating intensities of the two combined tints, the impression of one of the tints sometimes disappearing and reappearing....

"Binocular lustre was a species of lustre sui generis. It was a physiological, not a physical phenomenon, and had no 
relation whatever to those varieties of lustre which arose from the combination of lights reflected from the outer and inner surfaces of laminated, transparent, or translucent bodies. He assigned various causes for the physiological character of the phenomenon and then added, 'If binocular lustre arises from a physiological and not from a physical cause, we must look for this cause in the operations which take place in the eyes of the observer when binocular lustre is distinctly seen. These operations are of two kinds. First, in combining geometrical or other figures to represent solids whose parts are at different distances from the eye, the optic axes are in constant play, not only in varying the distance of their focus of convergence to unite similar points at different distances in the two diagrams, but in maintaining the unity of the picture by rapidly viewing every point of its surface. Secondly, when the two surfaces have different shades or colors, the retina of one eye is constantly losing and recovering the vision of one of them. Each optic nerve is conveying to the brain the sensation of a different tint or color. The brain is therefore agitated sometimes with one of these sensations and sometimes with both of them combined, and it is therefore not an unreasonable conclusion that, in the dazzle produced by this struggle of flickering sensations, something like lustre may be produced. In studying this subject of lustre there are some facts deserving of attention. In a daguerreotype for example, of two figures in black bronze with a high metallic lustre, it is impossible by looking at either of the pictures to tell the materials of which they are made. No lustre is visible; but when the two equally shaded pictures are combined in the stereoscope, the lustre and true character of the material is seen instantly. Another instructive example is seen in the stereoscopic representations of a boy blowing a soap-bubble. The lustre of the watery sphere is not visible in either of the two pictures; but when they are combined, it is seen distinctly. In both these cases and in others of the same kind, tints of similar intensity are combined, and there is not ground for assuming that the two surfaces combined appear at different 
distances, and the one is seen through the other as in Professor Dove's theory."

Rood $^{1}$ has made some very interesting experiments in connection with Dove's theory of lustre, in which Dove considers that externally reflected light in combination with internally reflected or dispersed light produces the sensation of lustre. This view of the nature of lustre opens the possibility of producing the individual lustre and appearance of gold, copper, brass, etc., by the stereoscopic combination of suitably colored surfaces. By combining tin-foil stereoscopically with yellow, orange, and ultramarine paper, Rood was able to produce the effect of gold, copper, and graphite, respectively.

Upon substituting dark gray paper for the tin-foil, the same effects in degree were not produced, owing apparently to the fact that the well-known texture and appearance of the paper forced itself so strongly on the attention as to preclude the sensation of a metallic surface. To get round this, a crumpled sheet of tin-foil was photographed and the positives from this gave almost as good a sensation of gold when combined with yellow paper as did the original tin-foil.

"Prof. Helmholtz, ${ }^{2}$ in his admirable work in physiological optics mentions that by a peculiar arrangement he was able to cause the homogeneous golden yellow light of the spectrum to appear brown, proving thus that the brown tint is only weak yellow light. These stereoscopic experiments give us on the other hand the means of apparently converting brown into metallic golden yellow, for many specimens even of brown wrapping paper, when combined in the stereoscope with very black prepared paper, acquire the lustre and appearance of yellow plates, in the shade and reflecting images of dark paper. In the same manner and corresponding to the investigations of Helmholtz, I found that the stereoscopic union of black glazed paper with red imitated with surprising perfection the appearance of a glazed plate of chocolate...

${ }^{1}$ Am. Jour. Sci., (2) 3I, I, 339 (I86r).

2 "Physiologische Optik," 28I (I 860). 
"I made some experiments to ascertain how far the stereoscopic mixtures of two masses of different colored light correspond to their true mixture by the method of rapid rotation, use being made of the imitations above described. It is, however, so difficult to compare a varying with a fixed tint that I will not record the results obtained; in many cases a certain moderate amount of agreement in the resultant tints was observed. Brücke found that, when a deeply colored yellow glass was held before one eye and a blue cobalt glass before the other, a landscape viewed through this combination was simply darkened in appearance. I repeated this experiment with similar glasses and obtained a like result; objects appeared darkened, but in their natural colors, though sometimes the blue or yellow tint predominated a little. But when I presented to a single eye these two masses of light a very different result was obtained; the plates of glass were attached to a blackened disc opposite suitable perforations, and it was set in rapid rotation; a landscape viewed through it appeared a deep purple, though not a trace of this color was to be perceived in the binocular use of these glasses. When these two glasses were held before the same eye, a landscape viewed through them was very much darkened but scarcely colored.

"Sir David Brewster opposes Dove's theory of lustre, as he has found that when black and white surfaces without drawings are combined in the stereoscope, no lustre is produced. The lustre, then, according to this philosopher is due not to one mass of light passing through another, but to the effort of the eyes to combine the stereoscopic pictures. Admitting the correctness of Sir David's experiments, Dove has shown that the objection founded on it is without weight. ${ }^{1}$

"In repeating Brewster's experiment I always obtain the opposite result; in combining uniform black and white surfaces, without drawings, I always obtain a distinct impression of lustre, like that of the blackened mirror of a polariscope, and, in strict accordance with Dove's theory, when the black

1 "Optical Studies," 3. 
field is so darkened that no light is sent from it to the eye, this lustre vanishes and the white paper alone is perceived. This disagreement is not a cause of astonishment when we reflect that de Haldat's original experiment waited nearly half a century for confirmation. To Brewster's own theory, the simple objection, which has already been made by others, that we daily perceive lustre plainly with one eye, would seem sufficient.

"I proceed now to describe some experiments where the idea of lustre is produced by the action upon a single eye of two masses of light of unequal intensity.

"I. If a disc of colored card-board, out of which a number of sectors has been removed, be made to rotate rapidly, and an object be viewed through it by a single eye, two masses of light will reach the eye, which apparently proceed from the object; one is reflected from the surface of the disc, the other emanates from the object behind the disc, and passes through the first mass of light. Dark objects viewed in this way assume to me to a small extent an appearance like that of blackened glass. The effect is not at all striking, and would be overlooked by many persons; I therefore prepared paper in a peculiar way so as to imitate distinctly the appearance of foliated graphite or crumpled mica.

"White smooth drawing paper was rubbed over irregularly with a brush slightly moistened with a weak wash of India ink or lampblack; when dry, another wash of a deeper hue applied as before, care being taken to leave many small spots untouched. The final wash was laid on with pure black. If the brush be kept nearly dry and passed only lightly over the paper, it is easy to obtain a surface bearing some very distant resemblance to the minerals above mentioned; it is of course without lustre. Similar papers were prepared with red and blue water colors.

"When these papers were held behind discs of ultramarine or orange-tinted paper, from which equal alternate sectors had been removed, and which were revolving at such rates that their surfaces seemed uniform, or at lower rates, 
they often appeared to a single eye highly lustrous. This was true of the prepared paper in a state of rest; when moved slightly by the hand it glittered strongly. Dark photographs of tin-foil held behind a revolving disc of ultramarine paper and viewed by a single eye, assume often to a striking degree the lustre and appearance of foliated graphite.

"2. If a piece of this peculiarly blackened paper $1 / 2$ of an inch square be placed in a blue field (rather light ultramarine paper), and be steadily regarded for some minutes by one eye it assumes a red-orange hue and appears suspended over the blue paper and nearer to the eye than the latter; at the same instant it appears lustrous like crumpled mica. The illusion with me often lasts half a minute in great perfection; this is particularly the case when the eye is not quite accurately focussed on the paper.

" 3 . If a sheet of this prepared paper be brightly illuminated by light from a window, and be held so near one eye as to produce indistinct vision, it often apparently becomes highly lustrous. In this case enlarged images of the white and gray points are formed on the retina which overlap, so that again we have two masses of light, one passing through the other.

"4. If a roll of black paper like the above, but coarser in its markings, be brightly illuminated on one side and viewed through deeply colored plates of glass (red, green, blue), in a few seconds it appears lustrous resembling a roll of polished zinc which has been irregularly and deeply corroded by an acid. Upon removing the glass the surface of the paper appears lustrous for an instant.

"5. A sheet of the finer variety of this prepared paper viewed through a large rhomb of calc spar gives often in spots the appearance of lustre, particularly when the head of the. observer, or the rhomb, is slightly moved. Some persons compared this to the appearance of water.

"It would seem probable that, in all cases where two masses of light reach a single eye, one passing through the other, particularly when there is any perception of their individuality, the appearance of more or less lustre is pro- 
duced, though from habit we often overlook it. Thus Helmholtz ${ }^{1}$ remarks upon the combination of two colored surfaces in monocular vision by means of a simple instrument he figures: 'It is particularly favorable when the drawings, or spots on the two surfaces are made to shift their position. Then we often believe that we see both colors simultaneously in the same place, the one through the other. We have an impression in such cases of seeing objects through a colored veil or reflected from a colored surface.",

"I found in fact that, by placing stereographs consisting of colored paper for one eye and a photographic drawing of tin-foil for the other in this instrument, lustre could be perceived, particularly with the imitations of copper....

"Analogous to this is the observation of Brewster" speaking of uniting similar pictures (patterns on hanging paper) in binocular vision, he remarks: 'The surface of it (the wall) seems slightly curved. It has a silvery transparent aspect.' Here the images, though of the same intensity, etc., moving with each slight movement of the head induce in the mind the idea of one object seen through another.

"In closing I will remark that while many of the experiments above mentioned are easily repeated, others require considerable practice in this kind of observation."

Brücke $^{3}$ has made an elaborate study of metallic lustre which must be included.

"Six gall-stones from the bladder of an ox had a surprisingly brilliant metallic lustre, so marked that one might easily have mistaken the concretions for an imitation gold alloy. I wrapped one of the stones in paper and struck it a light blow with a hammer. It broke into fragments of thin concentric layers. The inner and the outer surfaces were absolutely smooth and showed distinct metallic lustre. The thinnest of these pieces were transparent and the thicker ones opaque. I boiled one portion of the fragments in turpentine

1 "Physiologische Optik," 273 (x860).

2 "The Stereoscope," 9 I (1856).

${ }^{3}$ Sitzungsber. Akad. Wiss. Wien, 43, II, 177 (I86I). 
to replace the air films by a strongly refracting medium. They became more transparent and lost their metallic lustre. I embedded them in dammar varnish and examined them under the polarization microscope. They showed very distinct double refraction and with crossed Nicols showed partly the first order gray and partly colors of the higher orders. Here and there were to be seen small and very beautiful systems of rings with black crosses interspersed. Another portion of the fragments was dissolved in dilute acetic acid. Much carbon dioxide was given off and the filtrate contained calcium acetate and a little magnesium acetate in addition to free acetic acid. The part that was not dissolved by the acetic acid proved under the microscope to be organic matter which was brown in color and still kept the form of the fragments. It was very soft and without any signs of double refraction, was laminated, but showed no structure in the histological sense of the word. The concretion consisted therefore of this organic foundation, crystallized calcium carbonate and a little magnesium carbonate.

"In my collection I have gall-stones from another ox which are similar in form but only slightly lustrous. They are brown in color and have a scarcely perceptible metallic iridescence. Except for the lesser lustre they look like these cast zinc objects which are covered with a brown varnish to make them look like oxidized bronze. When broken they formed irregular fragments and not concentric layers. They were laminated however and this structure showed up clearly in the dark-colored organic material after treatment with acetic acid. They differed in chemical composition from the other samples by containing small amounts of phosphates.

"In Steiermark chamois are sometimes shot with teeth having a coating near the gums which glitters like gold. The hunters believe that in the inaccessible crevices among the rocks there are gold-bearing springs at which these animals quench their thirst. I have once had in my hands a lower jaw-bone with similar golden concretions on the teeth. The animal was shown by our late colleague, V. Kollar. I made 
no chemical experiments at the time but the laminated structure could be seen with the eye and most distinctly with a magnifying glass. I have no doubt but the material was the same kind of thing as the gall-stones.

"While studying these latter I wondered to what this very surprising metallic appearance could be due, and have therefore tried to find out what are the characteristics of metallic lustre and to what it is due. So long as one assumed that metals had very high indices of refraction, it was possible to ascribe the lustre to this, although it was not evident why one should get any sensation from the high optical density in view of the extreme opacity of the metals. Nowadays this assumption is untenable because the high indices of refraction were deduced from the angle of the polarization maximum and Cauchy has shown that entirely different results are obtained if one calculates the index of refraction from his wellestablished formulas. He finds the index of refraction of mercury to be $I .7$ which is smaller than that of spinel and only one-third of the value previously assumed.

"It is also impossible to deduce the metallic lustre from the elliptic polarization which is produced by the reflection from a metal of a plane-polarized ray whose plane of polarization is neither parallel with nor perpendicular to the plane of the incident light. Apart from the fact that in planepolarized light we see the rays discovered by Haidinger, the unaided eye gives no clue as to the polarization of light which reaches it. It is easy to show that the metallic effect is quite independent of the elliptic polarization by looking at a metal through a Nicol prism. In that case only plane-polarized light reaches the eye; but the metal still looks metallic. If one rotates the prism, the intensity of the lustre varies and to some extent the color itself but the general appearance remains metallic.

"We must therefore find other criteria which will establish a real connection with our optical sensations. With nonmetallic, lustrous substances the color of the light reflected at the surface is [practically] independent of the absorption 
color of the substance. The reflected light has [practically] the same color as the incident light. This fact has been known for a long time for single cases. Oersted discussed it with many illustrations and it was generally accepted. Later Arago found that the light corresponding to the absorption color was incompletely polarized perpendicularly to the incident plane. He concluded that this light came from the interior of the substance while the light giving the lustre was reflected from the surface and was polarized in the incident plane.

"If one looks at a colored lustrous surface in ordinary daylight-a colored gloss paper or a polished table top for instance-at a suitable angle through a Haidinger dichroscopic glass and turns it so that the main axis of the Iceland spar is parallel to the incident plane or makes an angle of $90^{\circ}$ with it, one has two images, one of which is nearly white and is due to the reflected light and one of which shows the absorption color. The experiment was first done in this form by Botzenhart ${ }^{1}$ and is well suited to make clear that in these cases the color of the lustre has nothing to do with the lustre. Metals behave differently, for the absorption color is determined by the color of the reflected light or, more properly speaking, the color we ascribe to a metal is that reflected by it when the incident light is white. Thus a red metal has a red lustre and a yellow metal a yellow one. If one repeats Botzenhart's experiment with a brilliantly colored metal, gold for instance, the two images are surprisingly different. The light polarized in the incident plane is much lighter and has a touch of red, while the other has a saturated golden yellow color. The difference in the color of the two images is still more striking in the case of copper. One might expect therefore to get the same effect as with non-metallic lustrous substances but less conspicuously because ordinary light is only polarized incompletely by metals even when it is below the angle of the polarization maximum. As a matter of fact the

\footnotetext{
1 Pogg. Ann., 68, $291(1846)$
} 
result of Botzenhart's experiments with metals is to be interpreted quite differently.

"The reflection of light from metallic surfaces has been studied so successfully by Sir David Brewster, MacCullagh, Cauchy, de Sénarmont and Jamin, that it is now one of the best-developed branches of optics and Jamin was able to put forward a theory of the colors of metals which rests just as directly on the general principles of the undulatory theory (as the explanation of refraction and diffraction colors) of Newton's rings, etc. From Cauchy's formula combined with Jamin's experimentally determined constants, it followed that colored metals change the color of reflected white light one way when the light is polarized in the incident plane and differently when the light is polarized perpendicularly to this plane, the color being more saturated in the first case and paler in the second case. ${ }^{1}$ Since we must consider ordinary light as a succession of oscillations with continuously varying azimuths, each one of which when reflected behaves like a train of waves polarized below the azimuth of the same amplitude and oscillation frequency, it is clear that we cannot get two images of the same color, when we break up the light reflected from the metal into light polarized in the incident plane and light polarized perpendicularly to it. But the light of the two images which we see in the dichroscopic glass is reflected light. This determines the color which we ascribe to the metal and the color of the metal is the color of the lustre. This close connection between color and lustre is recognized by the eye and causes us to give the term metallic lustre to non-metallic substances which show similar phenomena.

"If we are to make an exhaustive study of the characteristics of metallic lustre, there are one or two other points to be considered. The opacity of the metals is a point which is of the greatest importance in its bearing on the real cause of the phenomenon. We never see anything back of the

\footnotetext{
${ }^{1}$ Jamin: Ann. Chim. Phys., (3) 22, 3 I r (1848).
} 
surface of a metal, but merely what is reflected at or by the surface. We therefore cannot ascribe metallic lustre to any metallic substance which is not sufficiently opaque that we do not notice any transparency under ordinary conditions. In other words we get metallic lustre only when any light coming from the interior of the substance is masked completely by that reflected from the surface. Another point which characterizes metals is the intensity of the reflected light, even when the surface is not well polished. This is due in part to the high reflecting power and in part to the viscosity of the metals. A scratch on a brittle polished substance like glass or a gem, appears matt to the eye and so does a group of scratches. A scratch on a reguline metal is a lustrous groove.

"In order to get the full extent of metallic lustre one must see the surface as such. If a metallic surface is ground and polished very highly, and if one is some distance away, the surface disappears completely and one sees only one's own image. It is doubtful whether a man who had never seen such a mirror would recognize it as a metal plate without further test, even though he were otherwise familiar with metallic objects. If one sees the surface owing to scratches from grinding or to defective polishing, the character of the defective spots shows one at once that one is looking at a metallic surface. It can be shown that we get the impression of a metallic surface even with substances which have in themselves no metallic lustre, provided we have simultaneously a roughened surface and a high reflection of light. The most familiar instance of this is the silvery streak which marks the reflection of the moon from a water surface rippled by the evening breeze. The wavelets make the surface of the water seem rough and the accompanying high reflection of light makes the water look metallic, more metallic than the disc of the moon itself.

"I am now interested in showing that when we have metallic lustre with substances which are not metals, they satisfy the criteria which we have found to be necessary for 
metallic lustre. These criteria are the relation between lustre and color, relative opacity, and relatively high reflecting power even when the surface is not highly polished... One wellknown case of metallic lustre is that of total reflection. How does this happen? The intensity of the reflection or the amount of the reflected light resembles metallic reflection; but the illusion is complete only when no dioptically seen light comes from the reflecting surface to our eyes. The two other essential conditions are then fulfilled, that the reflecting surface appears to be that of an opaque body and that no light comes from the reflecting surface which can be distinguished by its color from that of the lustre and which might therefore seem to be the absorption color of the lustrous substance. Pour some water on a table near a window and place a beaker filled with water on the spilled water in such a way that there are air bubbles on the under side of the beaker. If one turns one's face toward the window and looks down at an angle through the water in the glass, the air bubbles look exactly like drops of mercury pressed out flat by the weight of the glass. This is much more striking than the better-known method of getting metallic lustre by dipping an empty test-tube into a cylindrical stand-glass containing water. The reflection at a surface does not have to be absolute. The same effect can be produced by a closely packed layer of superposed films. Thus exfoliated mica sheets will have a metallic lustre at an angle at which no single sheet would reflect totally, provided the sum total of the successively reflected light drowns out all the transmitted light.

"The pile of mica sheets suggests another group of illustrations, the metallic lustre of iridescent feathers. Why is the lustre of the peacock feathers, of the neck feathers of the pigeon, etc., so distinctly metallic? I have examined the feathers from the neck of a gray pigeon. The sheen is entirely on the outer surface and only on the edge of the feathers which are relatively firm and rigid, not where they are downy. When passing from perpendicular to grazing incidence the color changes from red to green or from green to red. If the 
feather is wetted, the colors disappear, reappearing when the feather dries again. It is best to use alcohol for this experiment because it is taken up more readily than water by the feather and evaporated more rapidly.

"Under the microscope with reflected light one can see the cells where the color appears. The dividing lines appear dark where two cells come together and one can also distinguish the nuclei. Since the dark pigment interferes with the microscopical examination by transmitted light, I took a white pigeon. The play of colors is of course much fainter on account of the large amount of white light; but the colors can be seen with the naked eye and are quite distinct under the microscope, where they are really quite beautiful, probably because with colors mixed with a great deal of white, the effect increases up to a certain point with decreasing intensity of light. When the dry feather is examined under the microscope, the colors of transmitted light are seen also though of course weaker than those of reflected light, just as happens with Newton's rings. The nuclei appeared as water-clear spots in their darker and colored surroundings, and the lines separating the adjacent cells also appeared bright; so that the parts which by reflected light were dark and colorless are now light and colorless. If the feather is moistened with alcohol, the darker parts become lighter and if the feather is soaked in turpentine and dammar varnish, everything becomes so clear and transparent that the boundaries of the cells are scarcely visible, instead of being light, colorless lines on a dark colored ground. In some places which had not been penetrated completely by the varnish, there were dark, mostly long-drawn out, microscopically small bubbles.

"This shows clearly that the colors on a pigeon's neck are the colors of thin films and are due to two reflections, one when the light passes from the light into the solid and the other when the light comes out from the solid into the air. The outer skin of the chameleon also shows iridescent colors and I have shown conclusively elsewhere, ${ }^{1}$ that they are

I Denkschriften Akad. Wiss. Wien, 4, I79 (1852). 
formed exactly as Newton's rings are. There the distance between the reflecting surfaces is very variable, so that the microscope always shows several colors simultaneously in the same cell. This is much less the case with the neck feathers of the pigeon, so that there is always one main color, with others appearing here and there. When examined under the microscope the important rays are those which are nearly perpendicular, in which case the predominant color is red by transmitted light and green by reflected light.

"Somewhat less definite results were obtained from a study of the tail feathers of the peacock, which are so beautiful in their iridescent colors and vividness of the metallic lustre. The dark brown pigment interfered with the microscopic examination and the tail feather of a white peacock showed no trace of iridescent colors. Depending on the way the light struck it, the position of the matt and the lustrous surfaces changed just as is the case with damask. In another point the peacock feathers differ markedly from those of the pigeon. They do not lose their colors when wetted, not even when boiled in turpentine, though the lustre and the tint are changed somewhat. For this reason it seems to me impossible that the iridescence of the peacock feathers can be due to a grating effect as with the iridescent buttons. I have found no trace of anything like that even with the highest magnification available. The theory of the iridescent buttons does not apply, but the theory of thin films does. If one places the so-called eye on the outer end of a peacock's feather between oneself and a source of light, horizontally on a support which turns on a vertical axis, it will be found that the colors remain the same during the rotation; but they change if the feather is raised or lowered. They are therefore independent of the direction of the light and vary with the angle of incidence. When passing from perpendicular to grazing incidence the green changes through blue to purple and copper-red changes to green.

"After these preliminary remarks on iridescent colors of feathers, we can discuss the properties to which they owe their 
metallic lustre. We find again the connection between lustre and color, which is of course a necessary consequence of the interference colors. There is also the opacity, the effect of which is quite noticeable, for the neck feathers of the white pigeon, though iridescent, show no trace of metallic lustre but only a sort of mother-of-pearl lustre. The third condition is also realized of high intensity of lustre and the degree of it in various parts of the feather determines the more or less metallic appearance. Quite apart from this, the copperred and golden yellow portions of the feathers seem more metallic than the other portions because we are familiar with red and yellow metals.

"Now let us take a couple of illustrations which seem not to confirm what I have said about the relation between color and lustre in a substance showing metallic lustre. This lack of agreement is apparent and not real. The natural red iron oxide, haematite, is red but its lustre is white or gray like that of iron and is metallic. We are apparently dealing with a metallic lustre, the color of which has nothing to do with the absorption color. The relation between the two is quite different from that in substances which do not show metallic lustre. If one looks at a lustrous portion of the surface of haematite with a dichroscopic glass, one does not see one white or gray image and a second which is red, as one does with red paper or red sealing wax. Both images are gray, one being lighter than the other. The difference is therefore that sealing wax and red paper are red when they are lustrous, whereas haematite is red only when the surface is matt and is not red but gray where it is lustrous. The opacity of the substance makes its lustre seem metallic and like the lustre of iron or of graphite.

"Indigo is another case of the same sort. Indigo is blue but its lustre is copper-red and metallic. When it shows a metallic lustre there is no sign of blue and when one looks through the dichroscopic glass at a reflecting surface polished with steel or agate, one sees two reddish images of slightly different tint as in the case of copper, but no blue image. 
The blue which we notice on the matt surface of indigo is an absorption color and is the same.color which very small particles of indigo show under the microscope by transmitted light. Indigo is blue only where single particles are so detached that light from one side may pass through the particle and be reflected at the other side. If the particles are pressed down by a steel polishing tool, the reflections disappear and consequently the blue. The surface is now lustrous and since no light comes from the interior owing to the great opacity of indigo, the surface appears copper-red.

"I will cite another case in which a lustre which is distinctly not metallic becomes metallic suddenly if one removes the light which was coming through the lustrous surface. I have a large colorless gall-stone of cholesterin which I sawed in two and polished the section in order to show the beautiful crystalline structure. If one looks at this through a Nicol prism below the polarizing angle, so that the main axis of the calc-spar is parallel with the plane of incidence, one sees the structure of the gall-stone very clearly because one is no longer disturbed by the light reflected from the surface. If one turns the prism $90^{\circ}$, the light coming from the interior disappears and with it the structure, while the lustrous surface with its slight irregularities and its scratches looks exactly like a ground and polished surface of iron.

"The dichroscopic glass of course registers both impressions simultaneously and adjacent in the two images. I have found the same phenomenon of an apparent metallic lustre in the image given by the light polarized in the incident plane at the ground surfaces of a diamond lying on a black ground and at the natural surfaces of a piece of cerussite in the royal mineral cabinet. This suggests an explanation of the so-called metallic-like lustre of demantoid (the green garnet usually sold as olivine). If a mineral has a very high power of reflection, this is a property in which it resembles both the metals and the diamond. If it transmits light, this is a property in which it differs from the metals. If the amount of transmitted light is not too large, it may be relatively small 
in comparison with that reflected from some of the surfaces, in which case these latter will appear actually metallic, while the absorption color may be noticeable at other surfaces. Consequently a mineral which seems to combine metallic with adamantine lustre will show more adamantine lustre, the more light it transmits, while it will look more metallic the less light it transmits. This is confirmed by three samples of red silver ore which Director Hörnes had the goodness to show me and in Mohs' mineralogy under red silver ore, I find the statement that demantoid lustre is common in the light forms (proustite) and semi-metallic lustre in the darker one (pyrargyrite).

"We can now take up a case in which the objective conditions for any lustre are not present and still less for metallic lustre; but where we think we see metallic lustre. My honored teacher, Professor Dove, showed some years ago that two projections of a body, for instance a pyramid, in which one surface was black and the other white, gave a solid image in the stereoscope with lustrous surfaces as though cut out of graphite. The question why we have any luster at all has been explained by Oppelt and Helmholtz as being due to the fact that one sees a light surface with one eye where one sees a dark surface with the other. One cannot put forward as an objection that it is possible to see the lustre of smooth objects with one eye or that Burckhardt ${ }^{1}$ has produced a sensation of lustre for one eye by certain combinations of white and black. This is only a suitable answer to those who consider that all lustre is due to unequal simultaneous impressions of corresponding surfaces on the two retinas. It is not a question of this but rather why in our special case we consider the relief that we see in the stereoscope as lustrous. When we look at matt surfaces, they appear to both eyes as equally illuminated. We therefore cannot conclude that the stereoscopic relief is due to matt surfaces, because we see white with one eye and black with the other. Lustrous surfaces are often dark to one eye and light to the other.

1 "Fortschritte der Physik," 3 Io (1854). 
In fact, if one looks at a lustrous surface from close up, the light and the dark are never distributed the same for the two eyes. It is therefore natural in our experiment to get the sensation of lustre when we see the same point dark with one eye and light with the other.

"It is easy to convince oneself how important is the contrast between the impressions upon the two eyes. If one takes the two projections of a four-sided, flattened pyramid and paints two of the opposite sides black leaving the other pair white in one image and reversing this in the other image, one sees a lustrous pyramid in the stereoscope; but it looks as though it were subject to a more or less changing and flickering lighting. This is the effect of the conflict between the corresponding portions of the retinas which are receiving opposing sensations. Depending on whether the sensation in one or the other retina gets the upper hand, in the brain, one or the other pair of surfaces appears the more strongly illuminated.

"This does not explain however why the lustre is metallic. Dove's comparison with graphite has been accepted by everybody. The lustre of graphite is called metallic by the mineralogists and we find the three criteria satisfied: high reflecting power, opacity, and lack of an absorption color distinct from the lustre. If we cut a piece of graphite with a knife, the surface has a metallic lustre but it is not smooth and does not reflect regularly. If we try to polish it, the natural graphite takes no real polish for small crystals break out under the buffing steel. If one examines such a surface under the microscope by reflected light, one notices that the reflecting facets send back white light of considerable intensity; but the others appear absolutely black. The distances between the two types of facets are so small however that to the naked eye the light from the bright facets appears to spread over the dark intervening spaces and consequently the graphite has a gray, lustrous appearance.

"If we go back to the stereoscopic phenomena, we see the surfaces lustrous because what we see dark with one eye we see light with the other; but we do not see them either as 
smooth or polished partly because there is no regular reflection and also because we see the more or less rough paper with one eye and the black, matt, surface of ink with the other. The black seen by one eye combines with the white seen by the other to give an alternately lighter or darker gray. We therefore see the surfaces gray and a gray which we cannot account for as gray pigment. Consequently we see the surface as somewhat rough and not polished, as lustrous and distinctly not matt, and since the sensations are nearest to those that we get with graphite and consequently we conclude that the surface looks like graphite. ${ }^{1}$

"In describing the experiment with the flattened foursided pyramids, I mentioned that the conflicts between the two fields of vision produced the impression of a flickering illumination. The more marked this effect the less continuous the metallic appearance and, in cases where the black predominated, I could persuade myself that the whole surface was covered with a glossy black paper. If one is to get the impression of metallic lustre, the impressions on the two eyes must blend to a gray. The reason for this is clear so soon as we ask ourselves why lustrous black substances do not have metallic lustre, even though they are opaque and show no absorption color differing from the color of the lustre. The third condition for metallic lustre is lacking. They do not show metallic lustre because they are black and therefore reflect too little light. If they reflected enough light to have metallic lustre, a smooth surface would send back light as polished steel does and therefore would not appear black. An uneven surface would also not appear black but lighter or darker gray according to circumstances, because the images on the retina of the single specular surfaces would reflect light enough to cover the dark intervening spaces by so-called irradiation, as happens with graphite.

"We have now analyzed metallic lustre in a number of cases in which it was observed in non-metallic substances,

${ }^{1}$ [It would hardly produce this effect upon people who had never seen lustrous graphite.-W. D. B.] 
including cases where the objective conditions for lustre were not present at all. We will now return to our starting point to the gall-stones with metallic lustre and will ask ourselves if they are similar to any of the cases studied. From what has been said as to their structure, it is clear that they are analogous to the exfoliated mica sheet. On account of the close series of reflections, they reflected back a great deal of light and were practically opaque because the light which was reflected twice from each layer could not penetrate deeply. The yellow color is due to the passage of the light through the colored organic ground. Since the colored light is specularly reflected, it is the color of the lustre even though in origin it did not differ from an ordinary absorption color. ${ }^{1}$ The gold color arose here just as it does in certain imitation gold frames which are first covered with silver foil to give the metallic lustre and then coated with a thin film of deeply colored varnish to change the white of the silver into the yellow of the gold."

This would be more satisfactory if Brücke had actually prepared a golden gall-stone. As it stands, it does not seem to me that Brücke has really made matters clear.

Helmholtz ${ }^{2}$ gives the following explanation of the phenomenon of metallic lustre when the two images in a stereoscopic picture are colored or illuminated slightly differently.

"When light falls on a matt surface, it is scattered uniformly in all directions and the surface appears equally bright from whatever angle it is viewed. Consequently, under normal conditions it appears equally bright to both eyes. Polished surfaces, on the other hand, give a more or less regular specular reflection. There may be a large number of larger or smaller, rounded protuberances. If these projections are polished so as to give surfaces predominating in one plane, incident light is reflected predominantly in the direction in which a perfect reflecting surface would send all

${ }^{1}$ [This seems to be reasoning in a circle.-W. D. B.]

2 "Handbuch der physiologischen Optik," 933 (1896). 
the light. It will often happen that one eye will be in line with the reflected light and the other not, in which case the one eye will see the surface more brilliantly lighted than the other will. When we look through at a stereoscopic picture, of which one image is brighter than the other, we receive the impression to which we are accustomed with polished surfaces but which we never get from a matt surface, and the picture looks polished. In a similar way, if a polished surface is surrounded by a colored one, the reflected light that reaches one eye may be colored differently from that which reaches the other eye, whereas a matt surface, under normal conditions, will appear colored uniformly. In a stereoscopic image, if one field is colored slightly differently from the other, this causes a sensation similar to that which a polished substance would produce. As a rule the color of the polished substance itself mingles with the two reflected colors, so that the difference in the colors reflected to the two eyes is ordinarily not great and consequently it is easier to produce the impression of lustre by combining two colors which are not very different, than by combining very lustrous but very different colors. In the latter case there is more effect of contrast than of lustre.

"According to Wundt's observations lustre can be obtained most easily by the combination of two colored fields when the two differ about equally from the background. When the contrast is great, one field or the other is apt to predominate too strongly... Dove's observation is important that when one or the other color predominates very much, the lustre disappears but reappears at the moment of change from one to the other when the two fields are seen simultaneously.

"Metallic lustre is characterized by the fact that the regularly reflected light is itself colored and not white as in the case of transparent substances. Metallic lustre is therefore to be seen with substances which give the colors of thin films, such as the brilliant feathers of some birds, and with substances like indigo which are very strongly colored and have high indices of refraction." 
It is not clear from this why we should not get the effect of metallic lustre by looking at suitable portions of the landscape through spectacles having two differently colored glasses. With a yellow and a blue glass in the spectacles one is combining two different images in much the same way as when one looks through a stereoscope at a blue and a yellow picture, and yet I am assured that no effect of metallic lustre can be obtained in this way.

Spring ${ }^{1}$ has published some experiments which seem to him to reconcile the views of Dove and Brücke. "Metals have a peculiar; indefinable lustre which is so characteristic that to many people the word metal connotes it. I do not mean by this that everything which shows a metallic lustre is a metal and still less that a metal cannot occur in a form where one sees no lustre. The statement is too broad and the converse is not always true. Besides there is nothing absolute about the word metal itself. Since metallic lustre is not a fundamental property of matter, one may ask what physical conditions give rise to it.

"Unless I am mistaken Dove' made the first attempt to account for the phenomenon. He had looked through a stereoscope at two images of a pigment, one colored blue and the other yellow. He expected to get an image in relief which would be colored green. To his great surprise the mixture of colors gave a glistening surface like that of polished steel. When he repeated the experiment by superposing a black and a white image he obtained the gray metallic lustre of lead or tin. Dove concluded that metallic lustre, or rather the shine, is always due to the reflection of light from two surfaces placed one behind the other. The focus of the eye being different for each color, the stereoscope cannot give a complete superposition of two images which are colored differently. In the case of a metal there are also produced two reflections of light, one from the true surface and the other from below the surface.

${ }^{1}$ Bull. soc. chim. Paris (2), 50, 2 I 8 (I888).

${ }^{2}$ Pogg. Ann., 83, 169 (1851). 
"This hypothesis postulates a definite transparency of metals, in fact quite a marked transparency because the reflections of light must take place from surfaces whose distance apart is of the order of the differences in the focal lengths of the eye for different colors. In addition Dove's hypothesis does not account for lustre on really transparent substances. The hypothesis is nevertheless generally accepted and Brewster $^{1}$ develops it further saying that metallic lustre is a subjective phenomenon due to the effort made by our eyes in accommodating themselves to the different colors. One other explanation has been offered, that by Briicke. ${ }^{2}$ According to him the color of the light reflected from the surface of a substance not having metallic lustre will be independent of the real color of the reflecting substance, while for the metals the color of the reflected light is that which we attribute to the metals, the incident light being white. Brücke mentions as another condition for metallic lustre the intensity of the reflected light, an intensity dependent on the opacity of the metals. He points out also that total reflection of light gives a complete imitation of metallic lustre.

"Brücke's theory is diametrically opposed to that of Dove, the first attributing to the opacity of metals what the second attributes to their transparency. I have made an observation which may perhaps help toward the solution of the question. It takes from the metallic lustre everything that is specific and thereby harmonizes the two theories.

"In the studies which I have made for ten years to see whether the properties characteristic of liquids and gases are to be found to some extent in solids, I have had occasion to submit a considerable number of substances to high pressures. The compression was carried out ${ }^{3}$ in a steel cylinder with polished inner walls. The powders to be compressed were always very fine and, so far as possible, they were prepared by chemical precipitation. This was always so for the

\footnotetext{
1 "Fortschritte der Physik," 8, 31 (1852).

2 Ibid, 17, 313 (I861).

${ }^{3}$ See Bull. Acad. roy. belg., (2) Ir, 9 (I880).
} 
sulphides, oxides and carbonates, and usually so for the salts and substances soluble in water.

"Some of the substances have formed cylinders with more or less marked metallic lustre, even when the powder was not that of a metal; others on the other hand formed cylinders having surfaces showing a glassy lustre which was more or less perfect, depending on the degree of agglomeration. For instance bismuth sulphide, copper sulphide and manganese dioxide showed a metallic lustre while zinc sulphide, mercuric oxide, copper carbonate, etc., appeared to be varnished on the surface. If the fine powders of substances belonging in these two categories are examined under the microscope by transmitted light, it is easy to see what is the controlling physical factor. Without exception the substances which show metallic lustre when compressed are opaque when powdered or at least appear so under the conditions of the experiment, while the other class of substances are more or less transparent in the form of fine powder.

"Metallic lustre therefore appears when a polished surface is formed from a sufficiently opaque substance. The more complete the opacity and the smoother the surface, the more pronounced is the metallic lustre. Since there is probably no substance absolutely opaque, there is probably also no substance absolutely transparent. It all depends on the thickness of the film. Between vitreous lustre and metallic lustre there may therefore occur all the intermediate stages which one can distinguish between transparency and opacity. According to this, metallic lustre does not depend in any way on the specific chemical nature of a substance but on its physical state. An allotropic form of a metal which was sufficiently transparent would have a vitreous lustre. This point of view harmonizes the theories of Dove and Brücke. Metallic lustre is produced when light is sent back from two reflecting surfaces (theory of Dove); but only when the two surfaces are so close together as to give almost total reflection (theory of Brücke):" 
Michelson ${ }^{1}$ has studied the special case of metallic lustre as observed in butterflies and beetles. "It has been abundantly proved that the usual 'flat,' 'dead,' 'uniform' coloring, brilliant as this sometimes can be, e.g., in birds, butterflies, and flowers, finds its simple explanation in the existence of pigment cells; so that the same cause (doubtless with many modifications) is here effective as in the great majority of cases previously considered. But the lively, variable 'metallic' glitter of burnished copper or gold; the reflection from certain aniline dyes; the colors of certain pigeons, peacocks, humming birds, as well as a number of butterflies, beetles, and other insects, require another explanation.....

"The designation 'metallic' at once suggests that there may be some common property of all these colors which is typified by the metals themselves. But, as is well known, the principal characteristic which distinguishes the metals from all other substances in regard to their action on light is their extraordinary opacity. A very important consequence of such opacity is that light is practically prevented from entering the substance at all, but is thrown back, thus giving the brilliant metallic reflexion so characteristic of silver, gold, copper, etc. In fact, the distance to which light can penetrate in most metals is only a small fraction of a light wave; so that a wave-motion such as constitutes light, strictly speaking, cannot be propagated at all. Again, as this opacity may be different for different colors, some would be transmitted more freely than others, so that the resulting transmitted light would be colored; and the reflected light would be complementary to the transmitted color. For most metals the difference is not very great; so that the reflected light, except in the case of gold and copper and a few alloys, is nearly white. In the case of the aniline dyes, however, there is a marked difference, as is clearly shown by their absorption spectrum. In transmitted light, even a very small thickness of fuchsine shows no yellow, green, or blue, and gives as a resultant of the remaining colors a beautiful crimson. The

1 Phil. Mag., (6) 2r, 554 (19r r). 
light which it reflects, however, is just this yellow and green which it refuses to transmit, and it accordingly shimmers with a metallic, golden green color, which changes when the surface is inclined, becoming full green or even bluish green when the illumination is sufficiently oblique. ${ }^{1}$

"The chief characteristics by which metallic reflexion may be distinguished may be summarized as follows:

"I. The brightness of the reflected light is always a large fraction of the incident light, varying from 50 percent to nearly roo percent.

"2. The absorption is so intense that metal films are quite opaque even when their thickness is less than a thousandth of a millimeter.

" 3 . If the absorptio nvaries with color, that color which is most copiously transmitted will be the part of the incident white light which is least reflected-so that the transmitted light is complementary to the reflected.

" 4 . The change of color of the reflected light has already been mentioned. It follows the invariable rule that the color always approaches the violet end of the spectrum as the incidence increases. If the color of the normal reflexion is violet the light vanishes (changing to ultra-violet), and if the normal radiation be infra-red it passes through red, orange, and yellow as the incidence increases.

"While the criteria just considered are the simplest and most convenient for general observation, it is to the more rigorous results of more refined optical methods that we must look for the final test of the quality of reflection in any given case; to determine whether or not a color phenomenon may be due to metallic reflection or to one of the other general causes. Such optical tests are furnished by the effect of reflection upon polarized light. The elements of the resulting elliptic vibrations may be expressed in terms of the

I The change in color is very much more marked when the light is polarized perpendicularly to the plane of incidence. As the angle of incidence approaches the angle corresponding to the "polarizing angle," the color is a deep blue or even purple. 
amplitude ratio $\mathrm{R}$ of the components, and of the phase difference $\mathrm{P}$ corresponding to the angle of incidence $\mathrm{I}$."

When $P$ and $R$ are plotted as ordinates against $I$ as abscissas, we get the phase curve and the amplitude curve, and "it is evident that metals have a smoother phase curve than semi-metallic substances like graphite and selenium, and these show less abrupt changes than do transparent substances such as glass and quartz. In fact we may take the steepness of the curve where it is steepest (better where the phase difference is $1 / 4$ ) as a measure of the transparency of the substance; and theory shows that this steepness is in fact proportional to the absorbing power of the substance......

"In the aniline colors the absorption varies enormously with the color, and we have all the gradations from metallic reflection to almost perfect transparence combined in a single specimen. The measurement of the phase change and the amplitude ratio for fuchsine and diamond green show changes in the form of the curves almost identical with those found for silver, steel, graphite, selenium, flint glass, crown glass, and quartz... The phase curve is much more characteristic in its changes than the amplitude curve. These specimens [of fuchsine and diamond green] are prepared by dissolving the aniline color in hot alcohol, filtering hot, and covering a hot glass surface with the solution. The alcohol evaporates rapidly leaving a mirror surface of a thickness of the order of a thousandth of a millimeter.

"A quite remarkable alteration occurs in the phase curves when the solution is diluted. The film deposited is very much thinner than before (from one-tenth to one onehundredth of the former thickness), and for some colors, the thickness is so small that considerable light is reflected from the surface of the glass. The resulting curve may then be negative, in this case for the colors red, orange, and yellow. Such a result has been predicted from theoretical considerations; ${ }^{1}$ but, so far as I am aware, no attempt has been made to show that this depends on the color of the incident light.

1 Drude: "Theory of Optics," 294. 
This, however, follows if we consider that the condition for such a negative phase curve is that the transition layer has an index of refraction greater than that of the second medium; and as the refractive index for magenta is low at the blue end of the spectrum and high at the red end, the inversion of sign is strictly in accord with the theory, of which indeed it furnishes a striking confirmation.

"On applying the simpler general tests of metallic reflection to the case of iridescent plumage of birds, scales of butterflies, and wing-cases of beetles, one is at once struck with the close resemblance these bear to the aniline colors in every particular:

"I. The intensity of the reflected light is much greater than for the 'non-metallic' plumage, etc.; in some cases approaching the value of the reflection factor of the metals themselves.

"2. The reflected light is always colored, showing either a rapid change of index of refraction, or of coefficient of absorption with the wave-length or color; and, indeed, it may perhaps be objected that these colors are far more vivid than any of the reflexion hues of the aniline dyes, or of any other case of 'surface color' hitherto observed.

" 3 . In the cases which could be investigated for this relation (unfortunately rather few) the transmitted light is approximately complementary to that which is reflected.

"4. The change of color with changing incidence strictly follows the law already mentioned - the color always changing toward the blue end of the spectrum as the incidence increases.

"This remarkable agreement has been pointed out by Dr. B. Walter in an admirable essay, 'Die Oberflächen- oder Schiller-Farben' and it is shown that none of the other causes of color phenomena (in particular interference and diffraction) can be effective; the laws which govern these last being totally different. It is therefore somewhat surprising to find that the contrary view is still held by many eminent naturalists, and it is hoped that the further evidence here presented may 
serve to emphasize the distinction between 'metallic' or 'surface' color and the remaining classes of color (due to pigments, interference, and diffraction).

"In attempting to apply the more rigorous optical test of the measurement of the phase-difference and the amplitude-ratios, one is met at the outset with the serious difficulty of the absence of true 'optical' surface. In fact, the materials we have to deal with (feathers, butterfly scales, beetle wingcases) are so irregular that the quantity of 'regularly' reflected light which is brought to a focus by the observing telescope is insignificant, and is often masked by the light diffusely reflected. But by the simple device of replacing the objective of the collimator and of the observing telescope by low-power microscope objectives of small aperture, these difficulties are so far removed that it has been possible to obtain results which compare favorably with those obtained with the aniline films. In some of the measurements it has been found possible to deal with a single butterfly scale; and in these the irregularities of the surface were often quite insignificant, or of such nature that they could be taken into account..... .

"The correspondence between the results of a set of measurements on a beetle having a lustre resembling that of burnished copper and of a set of measurements on a thin film of magenta is so remarkable that it leaves no room to doubt that in this case the metallic coppery color of the wingcase is due to an extremely thin film of some substance closely analogous in its optical qualities to the corresponding aniline dye. ${ }^{1}$ The thickness of the magenta film was not very accurately determined; but, from the fact it was deposited from a solution of $I / 20$ of the concentration of that which produced the corresponding thick film (whose thickness is about $0.005 \mathrm{~mm}$ ), it is estimated that the thickness of the film is of the order of $0.00025 \mathrm{~mm}$. It is, doubtless, unsafe to draw any more definite conclusion from this regarding

1 The character of the curves for the organic film is considerably more "metallic" than the corresponding curves for magenta. 
the film of the wing-case than to say that it is probably of the same order.

"An attempt was made to check this estimate by the following simple device. A portion of the ellipsoidal wing-case of mean radius $\mathrm{R}$ was removed by passing it very lightly over a piece of the finest emery-paper fastened to a flat piece of wood. This left a clean elliptical hole of mean radius $r$ showing the edges of the 'metallic' film whose width, $h$, could not be appreciated in a microscope with a half-inch objective. If this be estimated at less than $0.001 \mathrm{~mm}$ the relation $t / h=$ $r / \mathrm{R}=0.5 \mathrm{~mm} / 5 \mathrm{~mm}$ gives $t$, the thickness of the film, less than a ten-thousandth of a millimeter.

"A second specimen of the same general coppery lustre gave a set of curves which showed a double reversal; the phase-curve being positive for crimson and red, negative for orange and orange-yellow, and positive again for the yellow, green, and blue. A series of curves for a very thin film of magenta (estimated thickness $0.00005 \mathrm{~mm}$ ) gave results surprisingly resembling those of the beetle, the second point of inversion being, however, in the green instead of the yellow, and the 'metallic' character of the film being much less marked than in the beetle wing-case. The resemblance in the lower curves, showing the variation of maximum with the color, is even more striking. It can scarcely be doubted, therefore, that here again the metallic color is produced in a film whose thickness is of the order of a ten-thousandth of a millimeter or less....

"The beetle wing-cases furnish in many cases a fairly smooth surface, and the difficulties in obtaining the necessary measurements are far less than when working with feathers of birds or with butterfly scales. Nevertheless, the same general characteristics obtain in these, in both the phase-curves and the amplitude-ratios. The two curves do not always correspond; but it is probable that the difference may be explained by the difficulty in obtaining accurate results with surfaces so irregular. It is worthy of note that in all of these curves (except that furnished by a red humming-bird feather) the 
curves are negative; from which it is fair to conclude that the film which produces the surface color is very thin.

"The total number of specimens which have been examined is perhaps not so large as it should be to draw general conclusions, and it is clearly desirable that it be extended; but, so far, the evidence for surface film, as the effective source of the metallic colors in birds and insects; is entirely conclusive. It is clear that in all of these curves the descriptive color corresponds in general to that color for which the phase-curve is least steep and for which the amplitude curve is highest; and is complementary to the color for which the phase curve is steepest and the amplitude curve is lowest, as we should expect; since the former corresponds to high reflective power, while the latter is characteristic of transparent substances with but moderate reflecting power."

Some exceptions were found by Michelson." "The measurements of the phase-difference in the light reflected from the blue-winged butterfly (Morpho alga), instead of being zero at incidence, had values which ranged from +o.15 to - O.I5, and which were found to vary with the orientation of the specimen. There were also corresponding changes in the general character of the phase- and amplitude-curves, all of which showed clearly that the whole phenomenon is considerably complicated by a structure of the scales. An examination under the microscope revealed the presence of exceedingly fine hairs (which can only be seen in reflected light) arranged without much regularity with their length parallel with that of the scale. ${ }^{2}$ It was at first natural to attribute the blue color to the light diffracted from these hairs; and it is not impossible that some of the silky sheen which these butterflies exhibit is at least in part due to these hairs, whose diameter is much less than a light-wave, and which are therefore in the same relation to the light-waves

1 Phil. Mag., (6) 21, 564 (I9II).

2 There are three varieties of scales, of different shapes. These are arranged in overlapping layers, the outer layer being quite transparent and the lower one opaque. The middle layer is the one showing blue by reflection and brownish yellow by transmitted light. 
as the small particles which cause the blue color of the sky. But the changes in color with varying incidence, so characteristic of true 'surface colors,' were precisely the same in this specimen, and were practically independent of the orientation; whereas the changes with the angle of incidence, which should result on the hypothesis that the color is due to diffraction, should follow an entirely different law.

"Another species of butterfly (Papilio Ulysses) was also examined and found to yield normal 'surface-color' curves. There is in this case no such minute linear structure as in the case of Morpho alga; and as here the phenomenon is clearly a case of 'surface color,' so it is highly probable that the same cause is effective in the case of Morpho. Many other specimens were subsequently examined, but all fell into one or other of the two classes typified by these two.

"If a specimen of the beetle popularly known as the Diamond Beetle is examined with a low power under the microscope, the bright green dots on the wing-case are seen to consist of depressions from which spring brilliant and exquisitely colored scales; the colors varying throughout the range of the spectrum (green, however, predominating). The colors exhibited by these scales are so vivid and varied, and the changes so rapid with varying incidence, that it was at once evident that the effect must be due to diffraction from regular striations, which were accordingly looked for under a magnification of about rooo diameters. There were occasionally faint indications of striated structure, but so uncertain that if other indications had been less decided it might have been concluded that some other cause must have been effective. But on putting the microscope out of focus a moderately pure spectrum was observed and by measuring the angles of incidence and diffraction of the various colors, the 'grating' space could be determined, and was found to be of the order of a thousandth to a two-thousandth of a millimeter.

"The specimen was next examined by reflected light"

1 The observation is somewhat difficult on account of the very small working space when using high powers. 
and the striations at once appeared, the count of the striations giving numbers agreeing very well with the calculated values. Frequently a single scale showed two or even three series of striations, giving corresponding spectra in three different directions. Another important feature of these 'gratings' was shown in the fact that the light is all concentrated in a single spectrum, showing that the striations must have an unsymmetrical saw-tooth shape. ${ }^{1}$

"On immersing the specimen in oil or other liquid, little or no change is observed except in those specimens in which a small communicating aperture exists in the neck (point of support) of the scale. The oil can be seen to gradually fill the interior, and simultaneously all trace of color vanishes. ${ }^{2}$ It appears then that the color in this case is due to fine striations on the interior surface of the scale.

"Plustiotis resplendens is a beetle whose whole covering appears as if coated with an electrolytic deposit of metal with a lustre resembling brass. Indeed it would be difficult for even an experienced observer to distinguish between the metal and the specimen. On examination with the Babinet compensator, it was found that the reflected light was circularly polarized even at normal incidence, whether the incident light was polarized or natural. The proportion of circularly polarized light is greatest in the blue, diminishing gradually in the yellow portion of the spectrum, and vanishing in the orange-yellow-for which color the light appears to be completely depolarized. On progressing towards the red end of the spectrum, traces of circular polarization in the opposite sense appear, the proportion increasing until the circular polarization is nearly complete in the extreme red.

${ }^{1}$ It may be noted that the objection that the colors of birds and insects cannot be due to diffraction on account of the equalizing effects of the varying angles of incidence and diffraction, would not apply if the striations are so fine as to give practically a single spectrum extending over a range of $45^{\circ}$.

2 Sometimes a faint indication of color remains (usually greenish) which shows the characteristics of surface color. It is probable that the surface color acts conjointly with the effect of diffraction, and indeed the character of the spectrum indicates an excess of green which may thus be accounted for. 
"It was at first suspected that the phase difference (not always so great as one-quarter, but varying between 0.15 and 0.25) was due to linear structure, as in the case of Morpho alga; but on rotating the specimen about the normal no change resulted. The effect must therefore be due to a 'screw structure' of ultra-microscopic, probably of molecular dimensions. Such a structure would cause a separation of natural incident light into two circularly polarized pencils traveling with different speeds, and having different coefficients of absorption. Such cases have been observed in some absorbing crystals; but whereas in these the difference in absorption between the two circularly polarized pencils is quite small compared with the total absorption-here one of the two is almost totally reflected, while there is scarcely a trace of the other.

"If this hypothesis be correct, however, the selective absorption (or reflection) being reversed at the other end of the spectrum-then for the orange-yellow the resulting light should be compounded of these two; and the resulting light should be plane-polarized and not depolarized. The depolarization is in fact only apparent; for on using a moderately high power objective it is at once evident that there is a structure in the wing-case which causes a difference of phase between components varying very rapidly from point to point; and the resulting plane of the plane-polarized light varies with corresponding rapidity, leaving no trace of polarization when the observation is made with a telescope.

"The absorption coefficient for this specimen is quite of the order of that of the metals; and the thickness of the 'metallic' film is of the order of a ten-thousandth of a millimeter."

It will probably be simpler to consider the ordinary cases of metallic lustre before taking up the special case of binocular lustre, even though the two are intimately connected. Electrolytic silver is white and not metallic; polished silver is gray and metallic; while pulverulent silver is black and not metallic. The black of pulverulent silver is quite simple 
because the light is absorbed by multiple reflectors until no light comes out. As was shown by Wood, ${ }^{1}$ the same phenomenon can be obtained on a large scale with a bunch of polished steel needles. We get the sensation of white only when we have diffused reflection. With electrolytic silver the innumerable facets scatter the light in every direction, without absorption of particular rays, and the metal appears white. In the case of the polished metal we have a high reflecting power but the reflection is specular and relatively little comes to the eye unless one happens to be in the direct line with the light. In other words we have very low diffuse reflection and consequently the metal appears gray and not white. Since the metals are opaque and have high reflecting power for the rays which they absorb, all the reflection is from a space bounded by two surfaces very close together, the outer surface and the inner surface to which the light penetrates. This double reflection is always associated with metals and is therefore called by us metallic. We get the same phenomenon with such solids as magenta, and.we speak here of metallic lustre. In the case of total reflection at a glass surface we have high specular reflection and low diffuse reflection, so the surface appears gray. We are getting reflection from two surfaces close together and consequently we get the effect of a metal. We know that we are getting reflection from at least two surfaces because total reflection disappears completely if the film becomes too thin. ${ }^{2}$ With the colors of thin films we have reflections from two surfaces very close together and consequently they are metallic; we have interference and consequently the reflected light is colored. The colors of thin films are more intense and more metallic when there is a dark background which prevents other light from being mixed with the colors of the thin films.

While we are not conscious of the reflections from the two surfaces, it is probable that the eye takes them into account subconsciously and that we do get a slight but per-

\footnotetext{
I "Physical Optics," 449 (I9I I).

2 Ibid., 374 (I9II).
} 
haps continuous change of focus. If this is the case, we should expect to get the effect of a metal whenever we had similar adjustment of focus. Helmholtz has applied this point of view to the case of solid metals where he is laying stress more especially on the differences in adjacent portions of the surface. A given portion of the slightly irregular surface will appear bright to one eye and dark to the other. The adjustment of these two impressions gives rise to a flutter effect which is the physiological significance of metallic lustre.

In stereoscopic or binocular lustre ${ }^{1}$ the axes of the two eyes converge, while their normal position of repose is parallel. If the eyes get at all fatigued, they waver and do not hold their convergence. As soon as the error becomes noticeable, the brain brings the axes back again so that the pictures coincide once more. If the two pictures are colored or shaded differently, one eye will get tired before the other and the brain will momentarily give most of its attention to the second eye which is pointing in the right direction, while the first or fatigued eye will go more or less to sleep and will turn outward. After a short space of time, the second eye becomes tired and the first rested. The brain gives its attention now to the first eye, while the second turns outward. The result is that the brain gets a fluttering sensation superimposed on the perception of form and color. This flutter comes from the direction muscles and not from the retina. If the two pictures are of somewhat different brightness, the attention will shift from one to the other and the iris muscles will also flutter; the difference must not be too great or the brighter image will hold the attention all the time, so that there will be a more nearly steady state with less flutter. If the two pictures are of different colors there will be a flutter of color sensation through the optic nerve in addition to the flutter of the focusing muscles.

The impression of lustre is therefore due to the nervous flutter or unsteadiness of attention due to the brain from the

${ }^{1}$ I am indebted to Dr. Edgar Buckingham of the Bureau of Standards for this suggested interpretation of binocular lustre. 
directing muscles, the iris muscles, the focussing muscles, or the retina; or perhaps, in some cases, of all four. The cause of the flutter is a rapid shifting of the attention back and forth between the two eyes, probably as a result of fatigue. If fatigue is the cause, the eyes of different people would behave very differently. Some people would get the impression of binocular lustre when others would not. This seems actually to be the case, for Dove mentions that some of the phenomena are not very striking and many people do not see them. Brewster experienced no sensation of lustre when he combined in the stereoscope black and white surfaces having no geometrical form whereas Dove and Rood did perceive lustre under the same circumstances. Woolen goods striped black and white appeared lustrous to Burckhardt though they do not to most people. This seems to be an exaggerated case of the eyes wandering over the surface in a jerky manner.

Brewster says that lustre is due "to the effect of the eye in combining the two stereoscopic figures and to the dazzle occasioned by the alternating intensities of the two combined tints, the impression of one of the tints sometimes disappearing and appearing," which is practically the view advocated here though not put in quite so specific a form. In fact Brewster goes on to say that "the optic axes are in constant play, not only in varying the distance of their focus of convergence to unite similar points at different distances in the two diagrams, but in maintaining the unity of the picture by rapidly viewing every point of its surface." Where Brewster is wrong is in considering that binocular lustre has no relation to those varieties of lustre arising "from the combination of lights reflected from the outer and inner surfaces of laminated, transparent, or translucent bodies." Lustre is essentially a physiological or psychological phenomenon in all cases.

The more nearly the surface actually resembles a metallic surface, the easier it will be for the eyes to see it as metallic and the less fatigue will be necessary to produce the sensation of metallic lustre. This is brought out very clearly in Rood's experiments. Brücke points out "that we get the impression 
of a metallic surface even with substances which have in themselves no metallic lustre, provided we have simultaneously a roughened surface and a high reflection of light. The most familiar instance of this is the silvery streak which marks the reflection of the moon from a water surface rippled by the evening breeze. The wavelets make the surface of the water seem rough and the accompanying high reflection of light makes the water look metallic, more metallic than the disc of the moon itself."

Buckingham proposes distinguishing as follows between "glitter," "lustre," and "shine." When one looks at the moon as it is reflected from water that is not too smooth, one can distinguish the separate moving bright spots by looking carefully, and the effect is that of "glitter." If one looks farther out, there comes a point beyond which the separate moving bright spots are indistinguishable to the conscious mind, although they may still be there. This gives the impression of "lustre." From a quiet, clean surface of mercury or from a first-class optical mirror, one gets, not "lustre," but "shine." Shine is merely specular reflection. On this basis lustre is either an imperfect and irregular shine in which the irregularities are too fine to be noticed separately, or a fine-grained glitter-also too fine to be recognized consciously as composed of separate bright spots.

This seems to be a logical distinction. The only objection to it is that it excludes a mercury surface and the case of total reflection from being cases of metallic lustre and these are usually taken as our standards. On the other hand Brücke says: "In order to get the full extent of metallic lustre one must see the surface as such. If a metallic surface is ground and polished very highly, and if one is some distance away, the surface disappears completely and one sees only one's own image. It is doubtful whether a man who had never seen such a mirror would recognize it as a metal plate without further test even though he were otherwise familiar with metallic objects. If one sees the surface, owing to scratches from grinding or to defective polishing, the character 
of the defective spots shows one at once that one is looking at a metallic surface." The discrepancy between Buckingham's definition and the usual practice disappears if one speaks of metallic lustre only when one can see the mercury surface on account of dust, etc., or, in the case of total reflection, when the glass surface is not absolutely plane.

Buckingham raises an interesting point as regards the effect of spectacles with differently colored glasses. When looking at a landscape, the distance is so great that the eyes are pointed and focussed for infinity. Consequently a change of attention from one eye to the other does not cause any motion of the eyes, and consequently no special sensation of lustre. One might perhaps get an effect of lustre if one looked at an object closely-say 18 inches from the eyes, and it is also possible that some people might get an effect of lustre by looking even at a distant landscape. If they did, it would prove that a flutter of the directing muscles was not essential to the sensation of lustre. It might even turn out that the essential element was not a flutter of any particular set of muscles but a flutter of the attention. There is evidently room for a good deal of interesting experimenting here.

This hypothesis of Buckingham's regarding the production of the sensation of lustre seems to combine all the good points of the theories of Dove, Brewster, Rood, Brücke, Helmholtz, and Spring. It takes into account the possibility of great differences in individual observers, which has not been recognized specifically by the others, and it has the advantage as a working hypothesis of suggesting a great deal of research work which ought to be done.

Since metallic lustre of the ordinary type occurs when there is no light reflected back from any depth, we should get vitreous lustre when the transparency increases, which is what actually occurs. There is, therefore, no sharp dividing line between the two forms of lustre and there seem to be no quantitative experiments indicating at approximately what point most people would begin to differentiate between metallic and vitreous lustre. As a matter of fact it is very difficult 
to get people to commit themselves in any special case. Suppose one takes an electric fan with a light behind and a light in front. By varying the relative intensities of the two lights one can change from great opacity of the fan to great transparency and the lustre should apparently vary from metallic lustre to vitreous lustre. With an aluminum fan some people are willing to admit that this happens; but with a fan having brass blades, the color is so distinctive that most people insist that they see metallic lustre under all conditions. This seems to be an ideal case for a quantitative study of the difference between metallic and vitreous lustre.

It is not possible at present to give real definitions of the different types of lustre which have been distinguished by the people who write about gems; but the waxy lustre apparently occurs when we have a medium made turbid by a transparent phase. A suitable emulsion of oil in water is waxy. With increasing opacity it becomes opalescent. If too much fusible material is added in making porcelain, the mass becomes more transparent and we get a waxy porcelain. It is to be hoped that this whole question of lustre and of the types of lustre may receive more attention in the future than has been the case for a good many years. There seems to have been a wide-spread though erroneous opinion that we knew all about it.

Cornell University 